\title{
Development of High Yielding Cowpea [Vigna unguiculata (L.) Walp.] Lines with Improved Quality Seeds through Mutation and Pedigree Selection Methods
}

\author{
Elmahdy Metwally ${ }^{1}$, Mohamed Sharshar ${ }^{2}$, Ali Masoud ${ }^{3}$, Benjamin Kilian ${ }^{4}{ }^{\oplus}$, Shivali Sharma ${ }^{4}$, Ali Masry ${ }^{3}$, \\ Paul D. Shaw ${ }^{5}{ }^{\infty}$, Sebastian Raubach ${ }^{5}$, Atef Fiad ${ }^{3}$ and Mohamed Rakha ${ }^{1, *}$ \\ 1 Horticulture Department, Faculty of Agriculture, University of Kafrelsheikh, Kafr El-Sheikh 33516, Egypt; \\ elmahdy_metwally@agr.kfs.edu.eg \\ 2 Agricultural Engineering Unit, Kafr El-Sheikh Region, Kafr El-Sheikh 33516, Egypt; sharsharm940@gmail.com \\ 3 Vegetable Research Department, Horticulture Research Institute, Agriculture Research Center, Giza 12619, \\ Egypt; m_rakha1982@yahoo.com (A.M.); alimasry78@yahoo.com (A.M.); ma4297138@gmail.com (A.F.) \\ 4 Global Crop Diversity Trust, Platz der Vereinten Nationen 7, 53113 Bonn, Germany; \\ benjamin.kilian@croptrust.org (B.K.); shivalipbg@gmail.com (S.S.) \\ 5 Department of Information and Computational Sciences, The James Hutton Institute, Invergowrie, \\ Dundee DD2 5DA, UK; Paul.Shaw@hutton.ac.uk (P.D.S.); Sebastian.Raubach@hutton.ac.uk (S.R.) \\ * Correspondence: mohamed.rakha@agr.kfs.edu.eg; Tel.: +20-155-311-0804
}

Citation: Metwally, E.; Sharshar, M.; Masoud, A.; Kilian, B.; Sharma, S.; Masry, A.; Shaw, P.D.; Raubach, S.; Fiad, A.; Rakha, M. Development of High Yielding Cowpea [Vigna unguiculata (L.) Walp.] Lines with Improved Quality Seeds through Mutation and Pedigree Selection Methods. Horticulturae 2021, 7, 271. https://doi.org/10.3390/ horticulturae7090271

Academic Editor: Liwang Liu

Received: 12 July 2021

Accepted: 20 August 2021

Published: 30 August 2021

Publisher's Note: MDPI stays neutral with regard to jurisdictional claims in published maps and institutional affiliations.

Copyright: (c) 2021 by the authors. Licensee MDPI, Basel, Switzerland. This article is an open access article distributed under the terms and conditions of the Creative Commons Attribution (CC BY) license (https:// creativecommons.org/licenses/by/ $4.0 /)$.
Abstract: Cowpea [Vigna unguiculata (L.) Walp.] is a major legume crop and an important source of protein in Africa. The Kafr El-Sheikh University has a long history of cowpea breeding and improvement in Egypt. Two superior lines with high seed yield and quality were selected through mutation breeding and released to farmers as new varieties under the names Kafr El Sheikh-1 and Kaha-1. Crosses were made between these two varieties to further improve cowpea to meet farmers' demand. Using the pedigree selection method, 13 new superior $\mathrm{F}_{10}$ lines were selected and evaluated over 2 years for seed yield and related traits, earliness, and protein content under low $\left(16\right.$ plants $\left./ \mathrm{m}^{2}\right)$ and high $\left(24\right.$ plants $\left./ \mathrm{m}^{2}\right)$ plant densities. The results showed that plants grown in narrower space produced significantly higher seed yield per unit area than the plants grown in wider space. All developed lines produced significantly higher seed yield than the two parental lines in the 2018 trial and Kaha- 1 in the 2019 trial. Line number 6 proved to be the best genotype for earliness (73.5-73.9 days after sowing), seed yield (573-647 $\left.\mathrm{g} / \mathrm{m}^{2}\right)$, and crude protein content (22.7-24.3\%) in both trials. In addition, line 4 with bushy determinate growth habit and high seed quality was recently released as a new variety (Sakha-1). Several other cowpea lines have clear potential for release as new high-yielding varieties with early maturity and high seed quality for farmers in Egypt. Seeds of selected lines are available from Kafrelsheikh University. This shows that mutation breeding and pedigree selection methods are among the most promising breeding methods for cowpea improvement.

Keywords: field evaluation; plant density; protein content; legume crop; seed yield; Vigna

\section{Introduction}

Cowpea [Vigna unguiculata (L.) Walp.] is an important legume crop in developing countries, with $80 \%$ of production occurring in the dry savannas of tropical West and Central Africa [1]. Cowpea can be used in the form of dry seeds, fodder, green pod, green manure, and cover crops. It is mainly cultivated in Africa, including Egypt, for its dry seeds and/or green pods before maturity as a vegetable. Cultivated cowpea is a valuable source of protein, micronutrients, and vitamins [2]. This crop can grow in a wide variety of soils ranging from well-drained heavy clay to sand, as well as dry environmental conditions [3,4]. Cowpea can be used as intercrop with cereals, which improves soil fertility [5]. In addition, Rhizobia bacteria in root nodules of legumes fix atmospheric nitrogen at about $240 \mathrm{~kg}$ per 
ha and improve soil fertility and structure [6]. However, increasing abiotic stress such as drought, heat, and salinity, as well as high pressure from diseases and insect pests under climate change, reduce the yield and quality of existing legume varieties [7].

Besides abiotic and biotic stresses, plant density also plays an important role in determining the full seed yield potential of grain legume cultivars. The use of appropriate plant density is critical for optimum seed yield in legumes such as cowpea. There are several factors that affect plant density, such as soil moisture, available nutrients, crop management, and genotype [8]. In addition, the optimum plant density varies from region to region [9-13]. Cowpea varieties with different plant morphologies require different optimal densities to maximize seed yield and crop quality [14-16].

To date, genetic improvement of cowpea has lagged behind vegetable crops in Egypt. Production has been limited for many years to a few varieties that are relatively prostrate, late-maturing, and produce few seeds. A better understanding of legume diversity and the use of crop wild relatives in breeding can broaden the current narrow genetic basis in legumes including cowpea $[17,18]$. Mutation breeding, purification of already established varieties, and development of superior cowpea lines through pedigree selection are among the most promising breeding methods for cowpea. Resistance to cowpea aphids was reported in mutant lines (ICV 11 and ICV 12), which were expressed through antibiosis and antixenosis [19]. These mutant lines were obtained from ICV 1 seeds irradiated with $20 \mathrm{k}$ rad of gamma rays. The pedigree system of breeding is the most common method used by cowpea breeders, which has been successful in developing cowpea cultivars with new combinations of characteristics and resistance to diseases [12,20].

Through mutation breeding, two improved $\mathrm{M}_{5}$ lines were selected and released by Kafrelsheikh University and made available to farmers as the two best local cowpea varieties [Kafr El-Sheikh-1 and Kaha-1) at Kafr El-Sheikh region and then distributed throughout Egypt within a few years. Both varieties have bushy determinate growth habit and higher seed yield and quality than the old local variety Cream-7 [12]. In the present study, crosses were made between Kafr El-Sheikh-1 and Kaha-1 to increase genetic variability and select new cowpea lines with superior agronomic performance by pedigree selection. In addition, these lines were evaluated for earliness, seed yield traits, and crude protein to develop superior lines better adapted to climate change conditions. Several lines displayed early-maturing and higher seed yield than the parental varieties. These have clear potential for release as new varieties for farmers in Egypt. High yielding cowpea lines will be used to make crosses with wild Vigna species for development of pre-breeding lines for adaption to climate change.

\section{Materials and Methods}

\subsection{Development of New Cowpea Lines}

Cowpea seeds of variety Cream-7 were originally exposed to three doses of gamma radiation (10, 20, and $30 \mathrm{kr}$ ). Seeds were sown in April 1991 and 250 plants from each treatment were selected for $\mathrm{M}_{2}$ seed production. $\mathrm{M}_{2}$ plants were evaluated, and 25 mutant lines were selected and characterized by vigorous, bushy, determinate growth habit, high pod and seed yield, early maturity, and larger seeds than Cream-7. The mutant lines were evaluated during $\mathrm{M}_{3}$ and $\mathrm{M}_{4}$ generations, and the lines KFS-107 and KFS-61 were selected with the above superior traits. These lines were released in 1998 as new varieties for cultivation in Egypt under the names Kafr El-Sheikh-1 and Kaha-1 [12]. Crosses were made between Kafr El-Sheikh-1 as a female parent and Kaha-1 as male parent to produce $F_{1}$ seeds in the summer of 2006 . About $80 F_{1}$ plants were self-pollinated to produce $F_{2}$ seeds and then $2000 \mathrm{~F}_{2}$ plants were evaluated for the desired traits at three stages of plant growth: flowering, pod setting, and seed maturity. Selections for desired traits were repeated in subsequent generations from $\mathrm{F}_{3}$ until $\mathrm{F}_{10}$. A total of 13 promising improved lines were selected in the $F_{10}$ generation in 2016 and designated as lines 1, 2, 3, 4, 5, 6, 8, 9, 23, 28, 35,53 , and 56 . Overall, 13 promising cowpea lines were developed by pedigree selection 
according to Poehlman and Sleper [21] (Table 1) and evaluated under field conditions over two seasons.

Table 1. Breeding history of 13 cowpea lines derived from the crosses between the two local varieties Kafr El-Sheikh-1 and Kaha-1.

\begin{tabular}{|c|c|c|}
\hline Year & Generation & Breeding and Selection Activities \\
\hline 2006 & $\mathrm{~F}_{1}$ & Crosses between Kafr El-Sheikh-1 (female) and Kaha-1 (male) \\
\hline 2007 & $80 \mathrm{~F}_{1}$ plants & Grow $80 \mathrm{~F}_{1}$ plants to generate $\mathrm{F}_{2}$ seeds \\
\hline 2008 & $\begin{array}{l}2000 \mathrm{~F}_{2} \\
\text { plants }\end{array}$ & Select best individual $F_{2}$ plants \\
\hline 2009 & $\begin{array}{l}200 \mathrm{~F}_{3} \\
\text { plants }\end{array}$ & $\begin{array}{c}\text { Grow } F_{3} \text { plants, select the best rows within selected families, and } \\
\text { select best plants within selected rows }\end{array}$ \\
\hline 2010 & $\begin{array}{l}110 \mathrm{~F}_{4} \\
\text { families }\end{array}$ & $\begin{array}{c}\text { Grow } \mathrm{F}_{4} \text { lines, select the best rows within selected families, and } \\
\text { select best plants within selected rows }\end{array}$ \\
\hline 2011 & $\begin{array}{l}64 \mathrm{~F}_{5} \\
\text { families }\end{array}$ & $\begin{array}{c}\text { Grow } F_{5} \text { lines, select best rows within selected families, and select } \\
\text { best plants within selected rows }\end{array}$ \\
\hline 2012 & $35 \mathrm{~F}_{6}$ lines & Grow $\mathrm{F}_{6}$ lines, select the best families, and harvest best rows in bulk \\
\hline 2013 & $20 \mathrm{~F}_{7}$ lines & Grow $\mathrm{F}_{7}$ lines, select the best families, and harvest best rows in bulk \\
\hline 2014 & $13 \mathrm{~F}_{8}$ lines & $\begin{array}{c}\text { Testing } \mathrm{F}_{8} \text { lines and harvest best seed yield plots from one } \\
\text { replication in bulk }\end{array}$ \\
\hline 2015 & $13 \mathrm{~F}_{9}$ lines & $\begin{array}{c}\text { Testing } \mathrm{F}_{9} \text { lines and bulk best seed yield plots from one replication } \\
\text { to initiate pure seed development }\end{array}$ \\
\hline 2016 & $13 \mathrm{~F}_{10}$ lines & $\begin{array}{l}\text { Testing } \mathrm{F}_{10} \text { lines and bulk best seed yield plots from one replication } \\
\text { to increase pure seed }\end{array}$ \\
\hline
\end{tabular}

\subsection{Evaluation of Promising Cowpea Lines under Field Conditions}

Selected lines along with their parents, Kafr El-Sheikh-1 and Kaha-1, were evaluated in field trials during the 2018 and 2019 summer seasons for early maturity, seed yield, and crude protein content under two plant densities, i.e., 16 plants $/ \mathrm{m}^{2}(80 \mathrm{~cm}$ between ridges and $30 \mathrm{~cm}$ within rows) and 24 plants $/ \mathrm{m}^{2}(80 \mathrm{~cm}$ between ridges and $20 \mathrm{~cm}$ within rows) at the experimental farm, Faculty of Agriculture, Kafrelsheikh University (latitude $31^{\circ} 6^{\prime} 42^{\prime \prime} \mathrm{N}$, longitude $\left.30^{\circ} 56^{\prime} 45^{\prime \prime} \mathrm{E}\right)$. Mechanical and chemical analyses of the experimental soil are presented in Table 2.

Table 2. Mechanical and chemical analysis of the experimental field soil ( $0-30 \mathrm{~cm}$ depth) in the 2018 and 2019 trials.

\begin{tabular}{ccc}
\hline & & Trial \\
Variable & $\mathbf{2 0 1 8}$ & $\mathbf{2 0 1 9}$ \\
\cline { 2 - 3 } & & \\
Mechanical analysis & 10.00 & 9.20 \\
Sand\% & 32.40 & 31.90 \\
Silt\% & 57.60 & 58.90 \\
Clay\% & Clay & Clay \\
Textural class & & \\
Chemical analysis & 7.80 & 8.00 \\
pH & 3.31 & 3.30 \\
EC dsm-1 & 1.93 & 1.80 \\
Organic matter\% & 17.60 & 19.00 \\
Available N ppm & 7.60 & 7.70 \\
Available P ppm & 280.00 & 265.00 \\
Available K ppm & &
\end{tabular}

The evaluation trials were set up using a split-plot design with four replications. The two plant densities were arranged in the main plot and the cowpea genotypes (13 lines and two parental varieties) in sub-plots. Seeds were sown in hills using two plant densities of 16 plants $/ \mathrm{m}^{2}$ and 24 plants $/ \mathrm{m}^{2}$. The experimental plot $\left(8 \mathrm{~m}^{2}\right)$ contained one ridge of 
$10 \mathrm{~m}$ long and $0.8 \mathrm{~m}$ wide, and each hill had two plants. The recommended agricultural practices of the Egyptian Ministry of Agriculture were applied in both field trials in 2018 and 2019. Days to maturity was recorded as the number of days from sowing to maturity of $90 \%$ of the pods in each plot. Seed yield was determined by harvesting all pods in a 1 square meter area. The pods were threshed and weighed to obtain grain yield. Seed yield and its components were determined as seed yield $\mathrm{g} / \mathrm{m}^{2}$, number of pods $/$ plant, number of seeds/pod, seed index (weight of 100-seeds), and seed protein content. Nitrogen in the digested dry seeds was determined by the micro-Kjeldahl method according to Carter [22], and nitrogen in the dry seeds was multiplied by a factor of 6.25 to calculate the crude protein content.

\subsection{Statistical Analyses}

Statistical procedures were performed using SAS (version 9.1; SAS Institute, Cary, NC, USA). Days to maturity, seed yield, and crude protein data were subjected to analysis of variance (ANOVA) followed by a Duncan's Multiple Range test at the 0.05 probability level [23].

\section{Results}

The ANOVA showed significant differences among genotypes for all the traits in the 2018 and 2019 summer seasons (Figures 1 and 2). Significant differences were observed between plant density for seed yield and crude protein, and non-significant differences for days to maturity and number of pods per plant in both seasons (Table 3). Interaction between genotypes and plant density was significant for traits except for the number of pods per plant and number of seeds per pod in 2018, and days to maturity in the 2019 summer season (Table 3). This shows the differential response of genotypes for most of the traits under different plant densities.

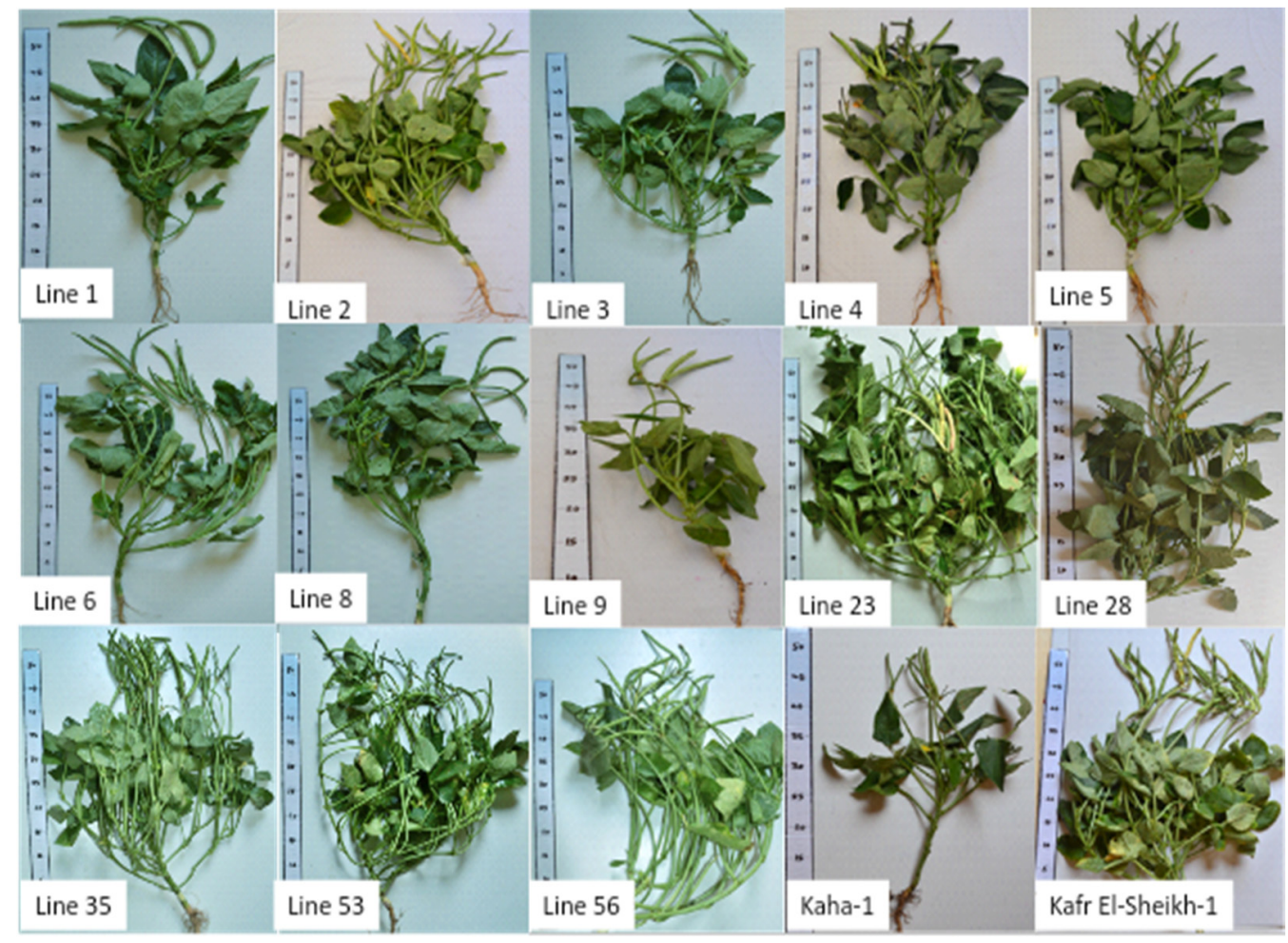

Figure 1. Overview of plant morphology of 13 selected $F_{10}$ lines compared to the parental lines Kaha-1 and Kafr El-Sheikh-1. 


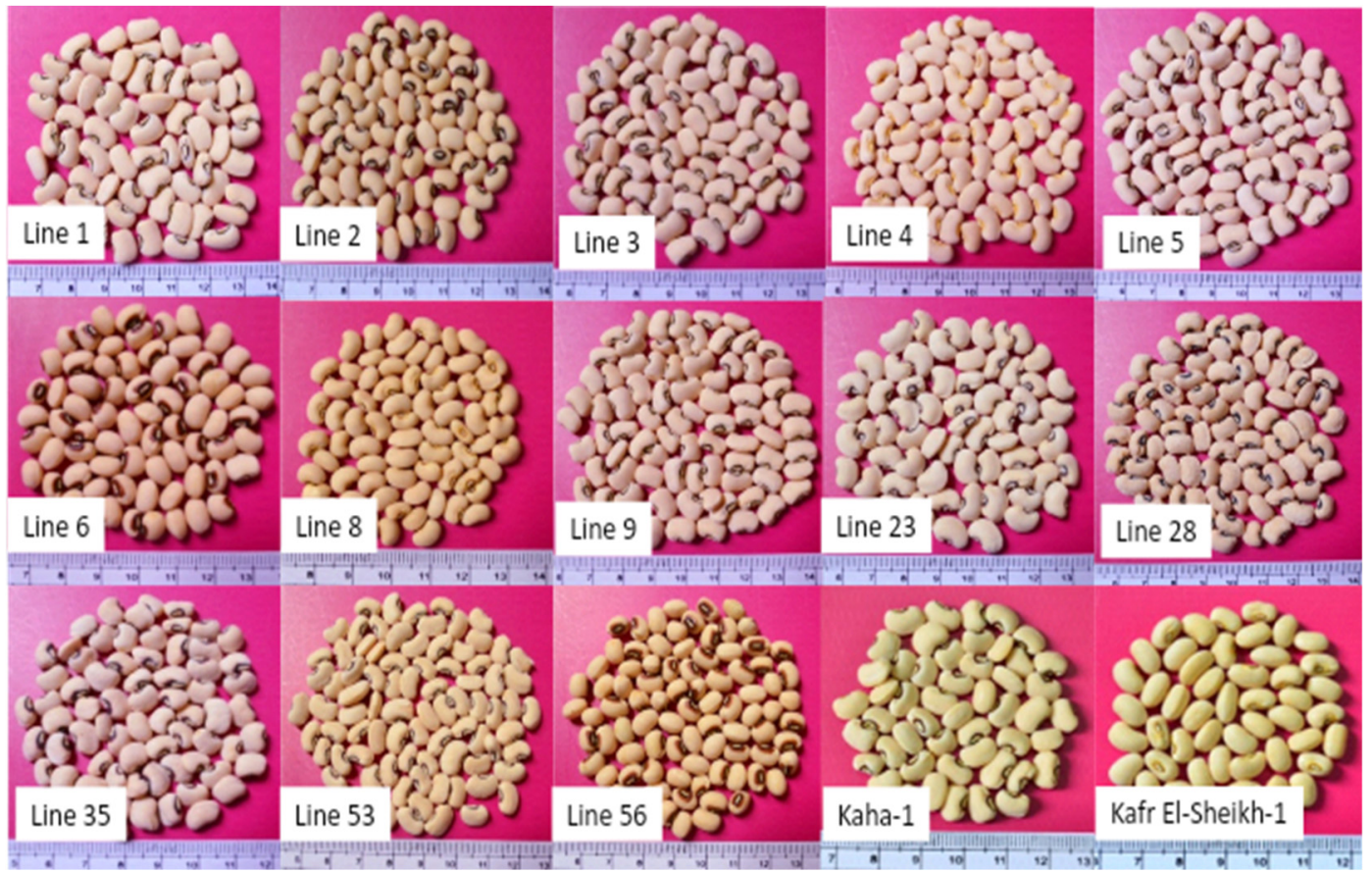

Figure 2. Seed size and color of 13 selected cowpea lines together with their parents Kafr El-Sheikh-1 and Kaha-1.

Table 3. Analysis of variance for different traits among 13 advanced breeding lines along with their parents evaluated under two plant densities (16 and 24 plants $/ \mathrm{m}^{2}$ ) for earliness, seed yield components, and crude protein during 2018 and 2019 summer season at Karf El-Sheikh region.

\begin{tabular}{|c|c|c|c|c|c|c|c|}
\hline \multirow[b]{2}{*}{ Source of Variation } & \multirow{2}{*}{$\begin{array}{l}\text { Degree of } \\
\text { Freedom }\end{array}$} & \multicolumn{6}{|c|}{ Mean Squares } \\
\hline & & Days to Maturity & $\begin{array}{l}\text { Seed Yield } \\
\left(\mathrm{Kg} / \mathrm{m}^{2}\right)\end{array}$ & $\begin{array}{c}\text { No. of } \\
\text { Pods/Plant }\end{array}$ & $\begin{array}{c}\text { No. of } \\
\text { Seeds/Pod }\end{array}$ & $\begin{array}{l}\text { Seed Index } \\
\text { (g/100 Seeds) }\end{array}$ & $\begin{array}{c}\text { Crude } \\
\text { Protein (\%) }\end{array}$ \\
\hline \multicolumn{8}{|c|}{2018 Summer Season } \\
\hline Replication & 3 & 0.5 & $29,614.47$ & 4.092 & 0.519 & 5.811 & 0.005 \\
\hline Plant density (A) & 1 & $0.033 \mathrm{~ns}$ & $830,003.30$ ** & $0.044 \mathrm{~ns}$ & $5.002 \mathrm{~ns}$ & $24.3^{* *}$ & $0.442 * *$ \\
\hline Error & 3 & 1.92 & 8210.097 & 5.353 & 2.292 & 0.122 & 0.001 \\
\hline Genotype (B) & 14 & $179.8^{* *}$ & $150,088.57^{* *}$ & $171.636^{* *}$ & $12.807^{* *}$ & $61.508 * *$ & $8.158^{* *}$ \\
\hline $\mathrm{A} \times \mathrm{B}$ & 14 & $10.64^{* *}$ & $6973.59 * *$ & $3.523 \mathrm{~ns}$ & $1.452 \mathrm{~ns}$ & $1.889^{* *}$ & $0.692 * *$ \\
\hline Error & 84 & 0.735 & 1335.29 & 3.969 & 1.01 & 0.104 & 0.001 \\
\hline Grand Mean & & 78.15 & 498.87 & 19.361 & 8.711 & 20.783 & 23.248 \\
\hline $\mathrm{CV}(\%)$ & & 1.1 & 7.32 & 10.29 & 11.54 & 1.55 & 0.16 \\
\hline \multicolumn{8}{|c|}{2019 Summer Season } \\
\hline Replication & 3 & 1.156 & 222.256 & 3.146 & 0.063 & 26 & 0.018 \\
\hline Plant density (A) & 1 & $2.133 \mathrm{~ns}$ & $\begin{array}{c}1,256,244.033 \\
* *\end{array}$ & $3.008 \mathrm{~ns}$ & $4.219 * *$ & $6.533 \mathrm{~ns}$ & $9.163^{* *}$ \\
\hline Error & 3 & 0.756 & 1874.522 & 2.521 & 0.173 & 16.044 & 0.011 \\
\hline Genotype (B) & 14 & $187.401^{* *}$ & $30,972.705^{* *}$ & $32.068^{* *}$ & $11.629^{* *}$ & $85.873^{* *}$ & $5.709^{* *}$ \\
\hline $\mathrm{AB}$ & 14 & $0.437 \mathrm{~ns}$ & $5079.676^{*}$ & $2.182 *$ & $0.207 * *$ & $4.301 * *$ & $2.694^{* *}$ \\
\hline Error & 84 & 0.527 & 2827.841 & 1.281 & 0.09 & 2.004 & 0.011 \\
\hline Grand Mean & & 80.633 & 483.383 & 18.2 & 8.232 & 19.433 & 23.104 \\
\hline $\mathrm{CV} \%$ & & 0.9 & 11 & 6.22 & 3.65 & 7.29 & 0.46 \\
\hline
\end{tabular}

* Significant at $p \leq 0.05 ;{ }^{* *}$ Significant at $p \leq 0.01 ; \mathrm{ns}=$ non-significant; $\mathrm{CV}=$ Coefficient of variation. 
As observed in ANOVA, the results presented in Tables 4 and 5 also showed that plant density had no significant effect on earliness in terms of days from sowing to $90 \%$ pod maturity in both 2018 and 2019 seasons. Conversely, genotypes had highly significant effects on earliness in both seasons. The parental lines reached $90 \%$ pod maturity at 81.0-81.8 days from sowing (Kafr El-Sheikh-1) and 78.9-79.5 days (Kaha-1) in the 2018 and 2019 seasons, respectively. Five new cowpea lines reached $90 \%$ of pod maturity earlier (65.4 to 77.3) than their parental lines. Regarding the interactions between plant density and genotypes for earliness, there were significant differences in the 2018 season (Tables 3 and 5). The results showed that line number 28 was the earliest in low and high plant densities, reaching maturity at 67.1 and 65.4 days in the 2018 and 2019 seasons, respectively (Table 5).

Table 4. Effect of plant density and genotypes on seed yield traits and crude protein of cowpea lines evaluated in 2018 and 2019 trials.

\begin{tabular}{|c|c|c|c|c|c|c|c|c|c|c|c|c|}
\hline \multirow[b]{2}{*}{ Factors } & \multicolumn{6}{|c|}{2018 Trial } & \multicolumn{6}{|c|}{2019 Trial } \\
\hline & $\begin{array}{l}\text { Days } \\
\text { to Ma- } \\
\text { turity }\end{array}$ & $\begin{array}{l}\text { Seed } \\
\text { Yield } \\
\left(\mathrm{g} / \mathrm{m}^{2}\right)\end{array}$ & $\begin{array}{l}\text { No. of } \\
\text { Pods/ } \\
\text { Plant }\end{array}$ & $\begin{array}{l}\text { No. of } \\
\text { Seeds/ } \\
\text { Pod }\end{array}$ & $\begin{array}{c}\text { Seed } \\
\text { Index } \\
\text { (g/100 } \\
\text { Seeds) }\end{array}$ & $\begin{array}{c}\text { Crude } \\
\text { Protein } \\
\%\end{array}$ & $\begin{array}{l}\text { Days } \\
\text { to Ma- } \\
\text { turity }\end{array}$ & $\begin{array}{l}\text { Seed } \\
\text { Yield } \\
\left(\mathrm{g} / \mathrm{m}^{2}\right)\end{array}$ & $\begin{array}{l}\text { No. of } \\
\text { Pods/ } \\
\text { Plant }\end{array}$ & $\begin{array}{l}\text { No. of } \\
\text { Seeds/ } \\
\text { Pod }\end{array}$ & $\begin{array}{c}\text { Seed } \\
\text { Index } \\
\text { (g/100 } \\
\text { Seeds) }\end{array}$ & $\begin{array}{c}\text { Crude } \\
\text { Protein } \\
\%\end{array}$ \\
\hline \multicolumn{13}{|c|}{ Plant density } \\
\hline 16 plants $/ \mathrm{m}^{2}$ & 78.2 & $415.7 \mathrm{~b}$ & 19.4 & 8.9 & $21.3 \mathrm{a}$ & $23.3 \mathrm{a}$ & 80.5 & $381.1 \mathrm{~b}$ & 18.4 & $8.4 \mathrm{a}$ & 19.7 & $22.3 \mathrm{~b}$ \\
\hline 24 plants $/ \mathrm{m}^{2}$ & 78.1 & $582.0 \mathrm{a}$ & 19.3 & 8.5 & $20.4 \mathrm{~b}$ & $23.2 \mathrm{~b}$ & 80.8 & 585.7 a & 18.0 & $8.0 \mathrm{~b}$ & 19.2 & $22.8 \mathrm{a}$ \\
\hline$F$-test & ns & $* *$ & ns & ns & $* *$ & $* *$ & ns & $* *$ & ns & $* *$ & ns & $* *$ \\
\hline 1 & $80.0 \mathrm{c}$ & $417.9 \mathrm{f}$ & $14.7 \mathrm{j}$ & $6.3 \mathrm{e}$ & $24.4 \mathrm{~b}$ & $23.1 \mathrm{i}$ & $79.6 \mathrm{~b}$ & $368.0 \mathrm{~d}$ & $20.7 \mathrm{a}$ & $6.3 \mathrm{f}$ & $23.5 \mathrm{ab}$ & $21.8 \mathrm{i}$ \\
\hline 2 & $70.4 \mathrm{~g}$ & $542.4 \mathrm{~d}$ & $18.0 \mathrm{~g}-\mathrm{i}$ & $8.2 \mathrm{~b}-\mathrm{d}$ & $18.6 \mathrm{j}$ & $23.1 \mathrm{i}$ & $70.9 \mathrm{e}$ & $461.5 \mathrm{c}$ & $15.2 \mathrm{~g}$ & $8.9 \mathrm{~b}$ & $18.3 \mathrm{f}$ & $22.0 \mathrm{gh}$ \\
\hline 3 & $77.3 \mathrm{e}$ & $565.4 \mathrm{~cd}$ & $18.0 \mathrm{~g}-\mathrm{i}$ & $7.3 \mathrm{~d}$ & $21.9 \mathrm{ef}$ & $23.5 \mathrm{e}$ & $79.9 \mathrm{~b}$ & $404.5 \mathrm{~d}$ & $18.7 \mathrm{~cd}$ & $9.7 \mathrm{a}$ & $23.9 \mathrm{a}$ & $22.1 \mathrm{~g}$ \\
\hline 4 & $83.8 \mathrm{a}$ & $430.9 \mathrm{f}$ & $20.9 \mathrm{de}$ & $8.1 \mathrm{~cd}$ & $20.1 \mathrm{i}$ & $24.4 \mathrm{~b}$ & $82.1 \mathrm{a}$ & $513.3 \mathrm{a}-\mathrm{c}$ & $15.9 \mathrm{~g}$ & $8.4 \mathrm{c}$ & $19.5 \mathrm{~d}-\mathrm{f}$ & $23.6 \mathrm{~b}$ \\
\hline 5 & $80.5 \mathrm{bc}$ & $473.4 \mathrm{e}$ & $18.3 \mathrm{f}-\mathrm{h}$ & $8.5 \mathrm{~b}-\mathrm{d}$ & $25.0 \mathrm{a}$ & $23.5 \mathrm{e}$ & $80.0 \mathrm{~b}$ & $478.8 \mathrm{bc}$ & $15.0 \mathrm{~g}$ & $7.8 \mathrm{e}$ & $22.5 \mathrm{ab}$ & $23.5 \mathrm{bc}$ \\
\hline 6 & $73.9 \mathrm{f}$ & $647.7 \mathrm{a}$ & $25.0 \mathrm{ab}$ & $10.8 \mathrm{a}$ & $20.9 \mathrm{~h}$ & $24.3 \mathrm{c}$ & $73.5 \mathrm{~d}$ & $573.0 \mathrm{a}$ & $16.2 \mathrm{fg}$ & $9.7 \mathrm{a}$ & $20.3 \mathrm{de}$ & $22.7 \mathrm{f}$ \\
\hline 8 & $81.1 \mathrm{~b}$ & $550.8 \mathrm{~cd}$ & $23.3 \mathrm{bc}$ & $8.0 \mathrm{~cd}$ & $15.5 \mathrm{~m}$ & $23.2 \mathrm{~g}$ & $79.8 \mathrm{~b}$ & $467.8 \mathrm{bc}$ & $17.3 \mathrm{ef}$ & $8.7 \mathrm{bc}$ & $13.4 \mathrm{~h}$ & $23.2 \mathrm{~cd}$ \\
\hline 9 & $81.0 \mathrm{~b}$ & $533.9 \mathrm{~d}$ & $20.3 \mathrm{ef}$ & 8.5 bcd & $23.0 \mathrm{c}$ & $23.5 \mathrm{e}$ & $80.1 \mathrm{~b}$ & $526.0 \mathrm{ab}$ & $18.4 \mathrm{c}-\mathrm{e}$ & $7.7 \mathrm{e}$ & $22.0 \mathrm{bc}$ & $22.9 \mathrm{e}$ \\
\hline 23 & $84.4 \mathrm{a}$ & $565.9 \mathrm{~cd}$ & 20. efg & $8.6 \mathrm{bc}$ & $22.1 \mathrm{de}$ & $22.8 \mathrm{j}$ & $82.5 \mathrm{a}$ & $468.8 \mathrm{bc}$ & $19.3 \mathrm{bc}$ & $6.7 \mathrm{f}$ & $20.0 \mathrm{de}$ & $22.2 \mathrm{~g}$ \\
\hline 28 & $67.1 \mathrm{~h}$ & $586.5 \mathrm{bc}$ & $22.7 \mathrm{~cd}$ & $10.5 \mathrm{a}$ & $21.5 \mathrm{~g}$ & 20.21 & $65.4 \mathrm{f}$ & $572.0 \mathrm{a}$ & $18.0 \mathrm{de}$ & $9.8 \mathrm{a}$ & $20.8 \mathrm{~cd}$ & $21.2 j$ \\
\hline 35 & $80.0 \mathrm{c}$ & $570.7 \mathrm{~cd}$ & $22.7 \mathrm{~cd}$ & $9.3 \mathrm{~b}$ & $21.1 \mathrm{~h}$ & $23.2 \mathrm{~h}$ & $80.0 \mathrm{~b}$ & $526.5 \mathrm{ab}$ & $20.3 \mathrm{ab}$ & $6.5 \mathrm{f}$ & $15.8 \mathrm{~g}$ & $21.9 \mathrm{hi}$ \\
\hline 53 & $76.6 \mathrm{e}$ & $622.5 \mathrm{ab}$ & $16.2 \mathrm{~h}-\mathrm{j}$ & $8.5 \mathrm{bc}$ & 16.31 & $23.7 \mathrm{~d}$ & $73.5 \mathrm{~d}$ & $491.3 \mathrm{bc}$ & $18.4 \mathrm{c}-\mathrm{e}$ & $8.1 \mathrm{~d}$ & $15.8 \mathrm{~g}$ & $22.2 \mathrm{~g}$ \\
\hline 56 & $76.4 \mathrm{e}$ & $554.6 \mathrm{~cd}$ & $26.4 a^{\prime}$ & $10.9 \mathrm{a}$ & $22.4 \mathrm{~d}$ & $24.6 \mathrm{a}$ & $76.0 \mathrm{c}$ & $528.8 \mathrm{ab}$ & $20.9 \mathrm{a}$ & $9.9 \mathrm{a}$ & $22.3 a-c$ & $24.3 \mathrm{a}$ \\
\hline Kaha-1 & $78.9 \mathrm{~d}$ & $120.5 \mathrm{~h}$ & $8.0 \mathrm{k}$ & $8.0 \mathrm{~cd}$ & $17.4 \mathrm{k}$ & $22.5 \mathrm{k}$ & $79.5 \mathrm{~b}$ & $378.3 \mathrm{~d}$ & $17.7 \mathrm{de}$ & $7.6 \mathrm{e}$ & $14.8 \mathrm{gh}$ & $22.0 \mathrm{gh}$ \\
\hline $\begin{array}{c}\text { Kafr } \\
\text { El-Sheikh-1 }\end{array}$ & $81.0 \mathrm{~b}$ & $300.0 \mathrm{~g}$ & $16.0 \mathrm{ij}$ & $9.3 \mathrm{~b}$ & $21.6 \mathrm{fg}$ & $23.4 \mathrm{f}$ & $81.8 \mathrm{a}$ & $492.5 \mathrm{bc}$ & 20.9 a & $7.8 \mathrm{de}$ & $19.0 \mathrm{ef}$ & 23.0 de \\
\hline$F$-test & $* *$ & $* *$ & ** & ** & ** & $* *$ & $* *$ & $* *$ & ** & $* *$ & $* *$ & $* *$ \\
\hline
\end{tabular}

** indicate significance at $p<0.0001$, respectively. Means with the same letter within the same column are not significantly different at $(p \leq 0.05)$ according to Duncan's multiple range test; each value represents the mean of four replicates.

Significant differences were observed between plant densities for seed yield (Table 3). Plants grown at high plant densities produced significantly higher seed yields per unit area $\left(24 / \mathrm{m}^{2}\right)$ than plants grown at low plant densities $\left(16 / \mathrm{m}^{2}\right)$ (Tables 4 and 5$)$. On the contrary, increasing plant density reduced the number of pods per plant, and seed index, thereby reducing the seed yield per plant.

The differences between genotypes were highly significant for seed yield and its components in both seasons (Tables 3 and 4). There were also significant differences between genotypes at low and high plant densities for seed yield in the 2018 trial (Table 5). Interestingly, all 13 cowpea lines produced significantly higher seed yield than parents in the 2018 trial, whereas only two lines ( 6 and 28) exhibited significantly higher seed yield than parents in the 2019 trial (Table 4). Line 6 had the highest seed yield in 2018 season and line 28 in 2019 season at low and high plant densities in both trials (Table 5). In addition, lines 6, 28, and 56 had the highest number of seeds per pod in both trials. Bold seeds (seed index) were found in lines 6 and 53 in the 2018 trial and lines 1, 3, 5, 9, and 56 in the 2019 trial. 
Table 5. Effect of interaction between plant density and genotypes on seed yield traits and crude protein in cowpea lines evaluated with their parents in 2018 and 2019 trials.

\begin{tabular}{|c|c|c|c|c|c|c|c|c|c|c|c|c|}
\hline \multirow{2}{*}{$\begin{array}{c}\text { Factors } \\
\text { (Genotype } \\
\text { x Plant } \\
\text { Density } \\
(\text { Plants } \\
\text { no. } / \mathrm{m}^{2} \text { ) } \\
\end{array}$} & \multicolumn{6}{|c|}{2018 Trial } & \multicolumn{6}{|c|}{2019 trial } \\
\hline & $\begin{array}{l}\text { Days to } \\
\text { Maturity }\end{array}$ & $\begin{array}{l}\text { Seed } \\
\text { Yield } \\
\left(\mathrm{g} / \mathrm{m}^{2}\right)\end{array}$ & $\begin{array}{l}\text { No. of } \\
\text { Pods/ } \\
\text { Plant }\end{array}$ & $\begin{array}{l}\text { No. of } \\
\text { Seeds/ } \\
\text { Pods }\end{array}$ & $\begin{array}{l}\text { Seed } \\
\text { Index } \\
\text { (g/100 } \\
\text { seeds) }\end{array}$ & $\begin{array}{l}\text { Crude } \\
\text { Protein } \\
(\%)\end{array}$ & $\begin{array}{c}\text { Days to } \\
\text { Matu- } \\
\text { rity }\end{array}$ & $\begin{array}{l}\text { Seed } \\
\text { Yield } \\
\left(\mathrm{g} / \mathrm{m}^{2}\right)\end{array}$ & $\begin{array}{l}\text { No. of } \\
\text { Seeds/ } \\
\text { Pod }\end{array}$ & $\begin{array}{l}\text { No. of } \\
\text { Pods/ } \\
\text { Plant }\end{array}$ & $\begin{array}{c}\text { Seed } \\
\text { Index } \\
\text { (g/100 } \\
\text { Seeds) }\end{array}$ & $\begin{array}{c}\text { Crude } \\
\text { Protein } \\
(\%)\end{array}$ \\
\hline $1 \times 16$ & $80.5 b-d$ & $348.0 \mathrm{k}$ & 13.8 & 6.3 & $24.3 \mathrm{~b}$ & $22.7 \mathrm{q}$ & 79.5 & $286.0 \mathrm{kl}$ & $6.3 \mathrm{op}$ & $21.6 \mathrm{a}$ & $23.0 \mathrm{ab}$ & $21.1 \mathrm{n}$ \\
\hline $2 \times 16$ & $70.0 \mathrm{i}$ & $472.8 \mathrm{gh}$ & 18.5 & 8.8 & $18.5 \mathrm{jk}$ & $23.3 \mathrm{~m}$ & 70.8 & $380.0 \mathrm{~h}-\mathrm{j}$ & $9.0 \mathrm{de}$ & $14.9 \mathrm{k}$ & $19.5 \mathrm{ef}$ & $21.7 \mathrm{~lm}$ \\
\hline $3 \times 16$ & $80.3 b-d$ & $464.8 \mathrm{gh}$ & 18.7 & 7.8 & $22.5 \mathrm{~d}$ & $23.1 \mathrm{n}$ & 79.8 & $335.0 \mathrm{j}-1$ & $10.1 \mathrm{a}$ & $18.9 \mathrm{~b}-\mathrm{f}$ & $23.5 \mathrm{ab}$ & $22.2 \mathrm{i}-\mathrm{k}$ \\
\hline $4 \times 16$ & $83.3 \mathrm{e}$ & $390.8 \mathrm{jk}$ & 20.2 & 8.5 & $20.8 \mathrm{gh}$ & $24.5 \mathrm{c}$ & 81.8 & $419.0 \mathrm{f}-\mathrm{j}$ & $8.6 \mathrm{e}-\mathrm{g}$ & $16.6 \mathrm{~g}-\mathrm{k}$ & $19.5 \mathrm{ef}$ & $23.4 \mathrm{de}$ \\
\hline $5 \times 16$ & $80.8 \mathrm{bc}$ & $400.8 \mathrm{i}-\mathrm{k}$ & 18.9 & 9.6 & $25.8 \mathrm{a}$ & $23.6 \mathrm{i}$ & 79.8 & $389.0 \mathrm{~g}-\mathrm{j}$ & $7.8 \mathrm{i}-\mathrm{m}$ & $15.1 \mathrm{k}$ & $21.5 \mathrm{~b}-\mathrm{e}$ & $22.5 \mathrm{hi}$ \\
\hline $6 \times 16$ & $73.3 \mathrm{~h}$ & 534.0 ef & 25.5 & 11.0 & $21.3 \mathrm{fg}$ & $24.1 \mathrm{f}$ & 73.5 & $480.0 \mathrm{ef}$ & $10.0 \mathrm{a}$ & $16.3 \mathrm{~h}-\mathrm{k}$ & $20.0 \mathrm{~d}-\mathrm{f}$ & $22.5 \mathrm{hi}$ \\
\hline $8 \times 16$ & $80.8 \mathrm{bc}$ & $439.2 \mathrm{~h}-\mathrm{j}$ & 22.7 & 8.3 & $16.3 \mathrm{~m}$ & $23.6 \mathrm{i}$ & 79.3 & 361.0 h-k & 8.9 def & $16.5 \mathrm{~g}-\mathrm{k}$ & $13.5 \mathrm{i}$ & $22.3 \mathrm{i}-\mathrm{k}$ \\
\hline $9 \times 16$ & 80.8 & $451.6 \mathrm{~g}-\mathrm{i}$ & 19.6 & 9.3 & $23.3 \mathrm{c}$ & $23.5 \mathrm{j}$ & 79.8 & $446.0 \mathrm{f}-\mathrm{i}$ & $8.0 \mathrm{i}-1$ & $18.9 \mathrm{~b}-\mathrm{f}$ & $21.5 \mathrm{~b}-\mathrm{e}$ & $22.4 \mathrm{ij}$ \\
\hline $23 \times 16$ & $84.3 \mathrm{a}$ & $468.8 \mathrm{gh}$ & 20.5 & 8.9 & $22.5 \mathrm{~d}$ & $23.3 \mathrm{~m}$ & 82.5 & $357.0 \mathrm{i}-\mathrm{k}$ & $7.3 \mathrm{n}$ & $19.3 \mathrm{~b}-\mathrm{e}$ & $20.0 \mathrm{~d}-\mathrm{f}$ & $23.0 \mathrm{fg}$ \\
\hline $28 \times 16$ & $68.0 \mathrm{j}$ & $478.8 \mathrm{f}-\mathrm{h}$ & 23.1 & 10.4 & $21.3 \mathrm{fg}$ & $20.2 \mathrm{u}$ & 65.3 & $430.0 \mathrm{f}-\mathrm{i}$ & $10.0 \mathrm{a}$ & $17.3 \mathrm{f}-\mathrm{j}$ & $21.5 \mathrm{~b}-\mathrm{e}$ & $21.6 \mathrm{~m}$ \\
\hline $35 \times 16$ & $80.5 b-d$ & $465.2 \mathrm{gh}$ & 21.5 & 9.0 & $20.8 \mathrm{gh}$ & $23.5 \mathrm{j}$ & 80.3 & $384.0 \mathrm{~h}-\mathrm{j}$ & $6.7 \mathrm{o}$ & $19.9 \mathrm{a}-\mathrm{d}$ & $16.5 \mathrm{gh}$ & 20.7 o \\
\hline $53 \times 16$ & $75.5 \mathrm{~g}$ & $512.4 \mathrm{e}-\mathrm{g}$ & 16.6 & 8.4 & 17.01 & $23.8 \mathrm{~g}$ & 73.0 & $396.0 \mathrm{f}-\mathrm{j}$ & $8.1 \mathrm{~g}-\mathrm{j}$ & $18.6 \mathrm{c}-\mathrm{f}$ & $16.5 \mathrm{gh}$ & $22.3 \mathrm{i}-\mathrm{k}$ \\
\hline $56 \times 16$ & $74.0 \mathrm{~h}$ & $455.2 \mathrm{~g}-\mathrm{i}$ & 27.0 & 10.8 & $23.5 \mathrm{c}$ & $25.0 \mathrm{a}$ & 76.0 & $375.0 \mathrm{~h}-\mathrm{j}$ & $10.0 \mathrm{a}$ & $21.6 \mathrm{a}$ & $22.5 \mathrm{a}-\mathrm{c}$ & $24.8 \mathrm{a}$ \\
\hline $\begin{array}{c}\text { Kaha-1 } \times 16 \\
\text { Kafr }\end{array}$ & 79.3 de & $104.8 \mathrm{~m}$ & 8.4 & 7.7 & $18.3 \mathrm{k}$ & $22.4 \mathrm{~s}$ & 79.8 & 275.01 & $8.1 \mathrm{~h}-\mathrm{k}$ & $21.6 \mathrm{a}$ & $16.5 \mathrm{gh}$ & $22.0 \mathrm{kl}$ \\
\hline $\begin{array}{l}\text { El-Sheikh-1 } \\
\quad \times 16\end{array}$ & $81.5 \mathrm{~b}$ & 248.41 & 15.8 & 9.1 & $22.8 \mathrm{~d}$ & $23.1 \mathrm{n}$ & 81.8 & $403.0 \mathrm{f}-\mathrm{j}$ & $7.7 \mathrm{j}-\mathrm{n}$ & $18.1 \mathrm{~d}-\mathrm{h}$ & $19.5 \mathrm{ef}$ & $22.8 \mathrm{gh}$ \\
\hline $1 \times 24$ & $79.5 \mathrm{c}-\mathrm{e}$ & $487.8 \mathrm{f}-\mathrm{h}$ & 15.5 & 6.2 & $24.5 b$ & $23.5 \mathrm{j}$ & 79.8 & $450.0 \mathrm{f}-\mathrm{h}$ & $6.4 \mathrm{op}$ & $19.8 \mathrm{~b}-\mathrm{e}$ & $24.0 \mathrm{a}$ & $22.5 \mathrm{hi}$ \\
\hline $2 \times 24$ & $70.8 \mathrm{i}$ & $612.0 \mathrm{~d}$ & 17.5 & 7.6 & $18.8 \mathrm{j}$ & $22.9 \mathrm{o}$ & 71.0 & $543.0 \mathrm{de}$ & 8.7 ef & $15.6 \mathrm{jk}$ & $17.0 \mathrm{gh}$ & $22.4 \mathrm{ij}$ \\
\hline $3 \times 24$ & $74.3 \mathrm{gh}$ & $666.0 \mathrm{~cd}$ & 17.4 & 6.9 & $21.3 \mathrm{fg}$ & $23.8 \mathrm{~g}$ & 80.0 & $474.0 \mathrm{e}-\mathrm{g}$ & $9.3 \mathrm{~cd}$ & $18.6 \mathrm{c}-\mathrm{f}$ & $24.3 \mathrm{a}$ & $22.1 \mathrm{jk}$ \\
\hline $4 \times 24$ & $84.3 \mathrm{a}$ & $471.0 \mathrm{gh}$ & 21.5 & 7.8 & $19.5 \mathrm{i}$ & $24.2 \mathrm{~d}$ & 82.5 & $607.5 \mathrm{~b}-\mathrm{d}$ & $8.3 \mathrm{~g}-\mathrm{i}$ & $15.3 \mathrm{k}$ & 19.5 ef & $23.8 \mathrm{c}$ \\
\hline $5 \times 24$ & $80.3 b-d$ & $546.0 \mathrm{e}$ & 17.7 & 7.3 & $24.3 \mathrm{~b}$ & 23.31 & 80.3 & $568.5 \mathrm{~d}$ & $7.8 \mathrm{j}-\mathrm{m}$ & $14.9 \mathrm{k}$ & $23.5 \mathrm{ab}$ & $24.4 \mathrm{~b}$ \\
\hline $6 \times 24$ & $74.5 \mathrm{gh}$ & $761.4 \mathrm{a}$ & 24.5 & 10.7 & $20.5 \mathrm{~h}$ & $24.5 b$ & 73.5 & $666.0 \mathrm{a}-\mathrm{c}$ & $9.5 \mathrm{bc}$ & $16.1 \mathrm{i}-\mathrm{k}$ & $20.5 c-f$ & $22.9 \mathrm{fg}$ \\
\hline $8 \times 24$ & $81.5 \mathrm{~d}$ & $662.4 \mathrm{~cd}$ & 24.0 & 7.8 & $14.8 \mathrm{o}$ & $22.8 \mathrm{p}$ & 80.3 & $574.5 \mathrm{~d}$ & $8.5 \mathrm{f}-\mathrm{h}$ & $18.1 \mathrm{~d}-\mathrm{h}$ & $13.3 \mathrm{i}$ & $24.2 \mathrm{~b}$ \\
\hline $9 \times 24$ & $81.3 \mathrm{~b}$ & $616.2 \mathrm{~d}$ & 21.0 & 7.6 & $22.8 \mathrm{~d}$ & $23.4 \mathrm{k}$ & 80.5 & $606.0 \mathrm{~b}-\mathrm{d}$ & $7.51-n$ & $17.9 \mathrm{e}-\mathrm{i}$ & $22.5 \mathrm{a}-\mathrm{c}$ & $23.6 \mathrm{~cd}$ \\
\hline $23 \times 24$ & $84.5 \mathrm{a}$ & $663.0 \mathrm{~cd}$ & 19.4 & 8.2 & $21.8 \mathrm{e}$ & $22.2 \mathrm{t}$ & 82.5 & $580.5 \mathrm{~d}$ & $6.0 \mathrm{~b}$ & $19.3 \mathrm{~b}-\mathrm{e}$ & $20.0 \mathrm{~d}-\mathrm{f}$ & $21.3 \mathrm{mn}$ \\
\hline $28 \times 24$ & $66.3 \mathrm{k}$ & 694.2 bc & 22.2 & 10.6 & $21.8 \mathrm{e}$ & $20.2 \mathrm{u}$ & 65.5 & $714.0 \mathrm{a}$ & $9.6 \mathrm{a}-\mathrm{c}$ & $18.6 \mathrm{c}-\mathrm{f}$ & $20.0 \mathrm{~d}-\mathrm{f}$ & 20.7 o \\
\hline $35 \times 24$ & $79.5 \mathrm{c}-\mathrm{e}$ & $676.2 \mathrm{c}$ & 23.9 & 9.7 & $21.5 \mathrm{ef}$ & $22.8 \mathrm{p}$ & 79.8 & $669.0 \mathrm{a}-\mathrm{c}$ & $6.3 \mathrm{op}$ & $20.7 \mathrm{ab}$ & $15.0 \mathrm{hi}$ & $23.1 \mathrm{e}-\mathrm{g}$ \\
\hline $53 \times 24$ & $77.8 \mathrm{f}$ & $732.6 \mathrm{ab}$ & 15.8 & 8.7 & $15.5 \mathrm{~m}$ & $23.5 \mathrm{j}$ & 74.0 & $586.5 \mathrm{~cd}$ & $8.1 \mathrm{~h}-\mathrm{j}$ & $18.2 \mathrm{~d}-\mathrm{f}$ & $15.0 \mathrm{hi}$ & $22.2 \mathrm{i}-\mathrm{k}$ \\
\hline $56 \times 24$ & 78.8 ef & $654.0 \mathrm{~cd}$ & 25.9 & 10.9 & $21.3 \mathrm{fg}$ & $24.1 \mathrm{e}$ & 76.0 & $682.5 \mathrm{ab}$ & $9.8 \mathrm{ab}$ & $20.2 \mathrm{abc}$ & 22.0 a-d & $23.8 \mathrm{c}$ \\
\hline $\begin{array}{c}\text { Kaha-1 } \times 24 \\
\text { Kafr }\end{array}$ & $78.5 \mathrm{ef}$ & $136.2 \mathrm{~m}$ & 7.6 & 8.3 & $16.5 \mathrm{~m}$ & $22.7 \mathrm{q}$ & 79.3 & 481.5 ef & $7.6 \mathrm{k}-\mathrm{n}$ & $17.4 \mathrm{f}-\mathrm{j}$ & $13.0 \mathrm{i}$ & $22.0 \mathrm{kl}$ \\
\hline $\begin{array}{l}\text { El-Sheikh-1 } \\
\times 24\end{array}$ & $80.5 b-d$ & $351.6 \mathrm{k}$ & 16.2 & 9.5 & $20.5 \mathrm{~h}$ & & 81.8 & $582.0 \mathrm{~d}$ & $7.5 \mathrm{mn}$ & $\begin{array}{c}20.2 a-c \\
f-j\end{array}$ & $18.5 \mathrm{fg}$ & $23.3 \mathrm{ef}$ \\
\hline$F$-test & $* *$ & $* *$ & ns & ns & $* *$ & $* *$ & ns & * & * & $* *$ & $* *$ & $* *$ \\
\hline
\end{tabular}

${ }^{*}$ and ${ }^{* *}$ indicate significance at $p<0.05$ and $p<0.0001$, respectively; ns = not significant. Means with the same letter within the same column are not significantly different at $(p \leq 0.05)$ according to Duncan's multiple range test; each value represents the mean of four replicates.

Crude protein content was significantly affected by plant density, genotypes, and their interaction in both seasons (Tables 3-5). Under low plant density, line 56 had the highest crude protein content in both trials (25\% in 2018 and $24.8 \%$ in 2019), whereas this line behaved differently under high plant density in 2018 and 2019 trials. Evaluation data of cowpea lines is available at: https:/ / ics.hutton.ac.uk/cwr/cowpea (accessed on 17 August 2021), which has allowed us to publish and make the data from this work available online. This has a number of advantages including facilitating unrestricted access of data to researchers, providing them with tools to explore and visualize their data, aligning with other cowpea data and allowing the downloading of data in standard formats. The two datasets that we have available for this work cover 11 different traits across 2018 and 2019 for 15 cowpea accessions and four replications. In total there are 2640 data points. The datasets can be visualized from within Germinate or downloaded using the following links: 2018 https://ics.hutton.ac.uk/cwr/cowpea/\#/data/export/trials/18 (accessed on 17 August 2021) and 2019 https:/ /ics.hutton.ac.uk/cwr/cowpea/\#/data/export/trials/19 (accessed on 17 August 2021).

\section{Discussion}

Cowpea is a major legume crop, which is rich in protein, carbohydrates, vitamins, and minerals, and complements the mainly cereal diet in countries that grow cowpea as a major food crop [1,2]. The old cowpea varieties in Egypt such as Cream-7 were late-maturing

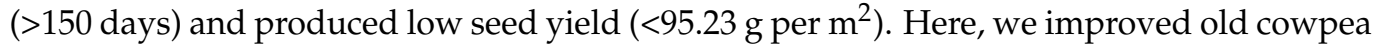


varieties and developed new high-yielding early-maturing cowpea lines that are preferred by farmers.

The results showed that five new cowpea lines were early and extra-early maturing lines (65 to 77 days), which reached $90 \%$ of pod maturity earlier than their parental varieties (Kafr El-Sheikh-1 and Kaha-1). This may be attributed to the fact that pod maturity in cowpea is more genetically controlled and less influenced by the environment [24]. Cowpea cultivars are generally classified into three categories in terms of pod maturity according to Ehlers and Hall [25]: extra-early ( $<60$ days from sowing to pod maturity), early (61-75 days from sowing to pod maturity), and late maturity ( $>80$ days from sowing to pod maturity). Harvesting in Egypt is done by handpicking the pods, and old varieties (indeterminate and prostrate) require three harvesting times or more. Our study developed new early-maturing cowpea lines that can be harvested in one time to save time and efforts.

In the present study, plants grown at high plant densities produced higher seed yields per unit area than plants grown at low plant densities. This may be attributed to the accommodation of a greater number of plants producing seeds per unit area under high plant density planting. Previous studies have also reported similar results wherein total yield was increased under high plant density [26-28]. On the contrary, increasing plant density reduced the number of pods per plant, and seed index, thereby reducing the seed yield per plant. This could be due to the increased competition among plants for available soil nutrients, moisture, light, and carbon dioxide [27], resulting in reduced translocation and accumulation of photosynthates from source to sink and thereby affecting overall plant growth and hence reduced seed yield per plant. Farmers in Egypt prefer to use high plant density (24 plants per meter square) for planting erect cowpea varieties. Newly developed 13 cowpea lines with bushy, determinate growth habit may require lesser inputs of water and fertilizer $[29,30]$ and are best suited for high-density planting as preferred by the farmers in Egypt.

Concerning seed yield, three cowpea lines exhibited significantly higher seed yield than parents in both trials. The components of seed yield in cowpea consist of three main traits, i.e., number of pods/plant, number of seeds/pod, and average seed weight. These components were superior in lines 6,28, and 53 compared to those in the other lines and original parents. In this context, Metwally et al. [30] indicated that mutation breeding could improve seed yield components of cowpea. In addition, Aliya and Makinde [24] reported that cowpea cultivars differ genetically in the expression of yield components, such as number of pods per plant, number of seeds per pod, seed index, and seed weight. Differences in seed yield traits among selected cowpea lines and parents in the 2018 and 2019 trials could also be attributed to environmental variations such as temperatures, light intensity, and humidity [12,30]. Interestingly, line 4 was released as new cowpea variety (Sakha-1) to farmers in Egypt in 2020. This variety has a bushy determinate growth habit, is early maturing, and has a high seed yield and protein content. Seeds of Sakha-1 have white hilum color and take a short cooking time, which are preferred traits by consumers.

Metwally et al. [29] indicated that there was no clear relationship between plant density and crude protein content in dry seeds of cowpea. In the present study, crude protein content was significantly affected by plant density, genotypes, and their interaction. Differences in the studies could be due to the use of different cowpea genotypes and/or differences in the growing environments. Nielsen et al. [31] also reported that cowpea cultivars differed in nutrient composition including protein content. Resistance to insectpests such as cowpea bruchids (Callosobruchus sp.), which cause significant losses in cowpea in Egypt, is still lacking in these lines. In general, the majority of cowpea losses is mostly attributed to biotic stress [32], in particularly insect-pests [33]. Resistance to insect-pests has been detected in wild Vigna species such as $V$. hirtella, V. minima, V. nepalensis, $V$. riukiuensis, $V$. tenuicaulis, $V$. umbellata, $V$. reflexopilosa ssp. glabra, and $V$. trinervia [17]. So far, no pestresistant varieties have been developed using these wild species because of the linkage drag of undesired traits and crossing barriers [34]. Further research is required for using wild 
relatives in cowpea breeding for developing biotic and abiotic resistant/tolerant varieties having better adaptation to climate change.

\section{Conclusions}

In the present study, 13 new superior lines with high seed yield, early maturity, and improved quality seeds were developed through mutation breeding and pedigree selection methods. Evaluation of these lines under different plant densities over seasons showed that these newly developed lines have higher yield potential than the parental lines and are the best suited for high-density planting in Egypt. Further, most of these lines are more early-maturing than parents, which makes them suitable for short-cropping seasons. It is interesting to note that one of these high-yielding lines, line 4 with high seed protein and bushy determinate growth habit, was recently released as a new variety, Sakha-1, in 2020. Further, line 6 having early maturity, high seed yield, and high crude protein content along with a few other high-yielding lines identified in the present study, has clear potential for release as new high-yielding varieties with early maturity and high seed quality for farmers in Egypt. Efforts will be made to introgress other useful traits such as insect-pest resistance into these lines by using wild species. Seeds of the new cowpea lines are available for seed distribution from breeders at Kafrelsheikh University, and evaluation data of cowpea lines is available through the Germinate platform [35,36] (https:/ /ics.hutton.ac.uk/cwr/cowpea (accessed on 17 August 2021).

Author Contributions: Conceptualization, E.M. and M.R.; methodology, E.M., M.R. and M.S.; validation, E.M. and M.R.; formal analysis, M.S., M.R., S.S., E.M., P.D.S., S.R. and A.M. (Ali Masry); investigation, E.M., M.R. and M.S.; resources, E.M. and M.R.; data curation, E.M., P.D.S., S.R., B.K., A.M. (Ali Masry) and M.R.; writing—original draft preparation, E.M. and M.R.; writing—review and editing, M.R., E.M., B.K., S.S. and A.F.; visualization, M.R.; supervision, E.M., A.M. (Ali Masoud) and M.R. All authors have read and agreed to the published version of the manuscript.

Funding: This work has been supported in part by the project "Evaluation and propagation of new superior lines from cowpea and garlic for releasing new varieties to farmers in Egypt" which is funded by the Academy of Scientific Research and Technology in Egypt http:/ / www.asrt.sci.eg/ (accessed on 17 August 2021) to release new cowpea varieties in Egypt. This work has also been supported in part by the project "Adapting Agriculture to Climate Change: Collecting, Protecting and Preparing Crop Wild Relatives" which is supported by the Government of Norway to make the evaluation data of cowpea lines available through the Germinate platform.

Data Availability Statement: The data presented in this study are available from the Germinate platform at: https:/ /ics.hutton.ac.uk/cwr/cowpea (accessed on 17 August 2021) or downloaded using the following links: 2018 https://ics.hutton.ac.uk/cwr/cowpea/\#/data/export/trials/18, (accessed on 17 August 2021) and 2019 https://ics.hutton.ac.uk/cwr/cowpea/\#/data/export/trials/ 19, (accessed on 17 August 2021).

Acknowledgments: This work has been supported in part by the project "Evaluation and propagation of new superior lines from cowpea and garlic for releasing new varieties to farmers in Egypt" which is funded by the Academy of Scientific Research and Technology in Egypt http: / /www.asrt.sci.eg/ (accessed on 17 August 2021), to release new cowpea varieties in Egypt. This work has also been supported in part by the project "Adapting Agriculture to Climate Change: Collecting, Protecting and Preparing Crop Wild Relatives" which is supported by the Government of Norway to make the evaluation data of cowpea lines available through the Germinate platform. The project is managed by the Global Crop Diversity Trust with the Millennium Seed Bank of the Royal Botanic Gardens, Kew UK and implemented in partnership with national and international genebanks and plant breeding institutes around the world (https:/ / www.cwrdiversity.org/ (accessed on 17 August 2021).

Conflicts of Interest: The authors declare no conflict of interest. 


\section{References}

1. Timko, M.P.; Ehlers, J.D.; Roberts, P.A. Cowpea. In Genome Mapping and Molecular Breeding in Plants; Kole, C., Ed.; Pulses, Sugar and Tuber Crops; Springer: Berlin/Heidelberg, Germany, 2007; Volume 3.

2. Foyer, C.; Lam, H.M.; Nguyen, H.; Kadambot, H.M.; Rajeev, K.V.; Timothy, D.C.; Wallace, C.; Helen, B.; Trevor, A.M.; Jonathan, M.; et al. Neglecting legumes has compromised human health and sustainable food production. Nat. Plants 2016, $2,16112$. [CrossRef]

3. Kay, D.E. Food Legumes; Tropical Development and Research Institute: London, UK, 1979; p. 32.

4. Hector, V.; Jody, S. Cowpea, Sustainable Agriculture Green Manure Crops; Cooperative Extension Service, University of Hawaii: Honolulu, HI, USA, 2002; SA-GM-6.

5. Dahmardeh, M.A.; Ghanbari, B.; Syahsar, A.; Ramrodi, M. The role of intercropping maize (Zea mays L.) and Cowpea (Vigna unguiculata L.) on yield and soil chemical properties. Afr. J. Agric. Res. 2010, 5, 631-636.

6. Aikins, S.H.M.; Fuakwa, J.J.A. Growth and dry matter yield responses of cowpea to different sowing depths. J. Agric. Biol. Sci. 2008, 3, 50-54.

7. Scheelbeek, P.F.; Frances, D.; Bird, A.; Tuomisto, H.L.; Rosemary, G.; Francesca, B.H.; Edward, J.; Joy, M.; Chalabi, Z.; Allen, E.; et al. Effect of environmental changes on vegetable and legume yields and nutritional quality. Proc. Natl. Acad. Sci. USA 2018, 115, 6804-6809. [CrossRef]

8. Eric, B. Traditional Field Crops, (Peace Crops)-Appropedia: The Sustainability Wiki. 1981, p. 283. Available online: https://www. appropedia.org/Traditional_Field_Crops (accessed on 9 July 2021).

9. Rubaihaya, P.; Radely, R.W.; Khan, T.N.; Mukubi, J.; Leakey, G.L.; Ashley, G.M. Nutritional Improvement of Food Legumes by Breeding; Protein Advisory Group of the United Nations System: New York, NY, USA, 1973; UN System.

10. Eny, B.A.C. A spacing/time of planting trail with cowpea [Vigna unguiculata (L.) Walp.]. Exp. Agric. 1974, 10, 87-95.

11. Ojehomon, O.O.; Bamiduro, T.A. The effect of plant density and pattern of plant arrangement on cowpea [Vigna unguiculata (L.) Walp.] using parallel row systematic spacing design. Field Crop. 1971, 27, 646.

12. Metwally, E.I.; Hewedy, A.M.; Hafez, M.; Morsy, M.A. Kafr El-Sheikh-1 and Kaha-1 new cultivars of cowpea. J. Agric. Sci. Mansoura Univ. 1988, 23, 3887-3897.

13. Allaa-Shaban, M.I. Application of Bio-Fertilizers to Fertilize Cowpea Plants under El-Arish Region Conditions. Master's Thesis, Arish University, North Sinai, Egypt, 2018.

14. Ndiaga, C. Genotype $x$ row spacing and environment interaction of cowpea in semi-arid zones. Afr. Crop. Sci. J. 2000, 9, 359-367.

15. Mawo, Y.M.; Mohammed, B.; Garko, M.S. Effect of phosphorus levels on growth, yield and development of cowpea (Vigna unguiculata (1.) Walp) varieties. Int. J. Sci. Eng. Appl. Sci. 2016, 2, 302-312.

16. Oroka, F.O. Mineral fertilizer and inter-row spacing effects on vegetative growth, nodulation and dry matter yield of cowpea (Vigna unguiculata L. walp). Int. J. Agric. Rural. Dev. 2017, 20, 3066-3073.

17. Van Zonneveld, M.; Rakha, M.; Tan, S.Y.; Chou, Y.-Y.; Chang, C.-H.; Yen, J.-Y.; Schafleitner, R.; Nair, R.; Naito, K.; Solberg, S. Mapping patterns of abiotic and biotic stress resilience uncovers conservation gaps and breeding potential of Vigna wild relatives. Sci. Rep. 2020, 10, 2111. [CrossRef]

18. Kilian, B.; Dempewolf, H.; Guarino, L.; Werner, P.; Coyne, C.; Warburton, M.L. Crop Science special issue: Adapting agriculture to climate change: A walk on the wild side. Crop Sci. 2021, 61, 32-36. [CrossRef]

19. Arturo, G.; Jens, W.; Jan, P. Cowpea aphid performance and behaviour on two resistant cowpea lines. Entomol. Exp. Appl. 1988, $49,259-264$.

20. Obisesan, I.O. Evaluation of pedigree and single seed descent selection methods for cultivar development in cowpea (Vigna unguiculata L. Walp). Plant Breed. 1992, 108, 162-168. [CrossRef]

21. Poehlman, M.; Sleper, D.A. Breeding Field Crops; Iowa State University Press: Ames, IA, USA, 1995; p. 495.

22. Carter, M.R. (Ed.) Soil Sampling and Methods of Analysis; Lewis Publishers: Boca Raton, FL, USA, 1993.

23. Duncan, B.D. Multiple range and multiple F test. Biometrics 1955, 11, 1-42. [CrossRef]

24. Aliyu, O.M.; Makinde, B.O. Phenotypic analysis of seed yield and yield components in cowpea [Vigna unguiculata (L.) Walp.]. Plant Breed. Biotechnol. 2016, 4, 252-261. [CrossRef]

25. Ehlers, J.D.; Hall, A.H. Cowpea [Vigna unguiculata (L.) Walp.]. Field Crop. Res. 1997, 53, 187-204. [CrossRef]

26. Njoku, D.N.; Muoneke, C.O. Effect of cowpea planting density on growth, yield and productivity of component crops in cowpea/cassava intercropping system. Agro-Science 2008, 7, 106-113. [CrossRef]

27. El-Naim, A.M.; Jabereldar, A.A. Effect of plant density and cultivar on growth and yield of cowpea [Vigna unguiculata (L.) Walp.]. Aust. J. Basic Appl. Sci. 2010, 4, 3148-3153.

28. Kamara, A.Y.; Tofa, A.I.; Kyei-boahen, S.; Solomon, R.; Ajeigbe, H.A.; Kamai, N. Effects of plant density on the performance of cowpea in Nigerian savannas. Exp. Agric. 2018, 54, 120-132. [CrossRef]

29. Metwally, E.I.; Moustafa, S.M.; Mazrouh, A.Y.; Fayed, A.M. Effect of genotypes, plant density and fertilizer level on seed yield and its components of cowpea. J. Agric. Res. Tanta Univ. 1998, 24, 237-246.

30. Metwally, E.I.; El-Waraky, Y.B.; Masoud, A.M.; Kasem, M.H. Developing and evaluation of some superior lines of cowpea. Alex. J. Agri. Res. 2012, 57, 273-280.

31. Nielsen, S.S.; Brandt, W.E.; Singh, B.B. Genetic variability for nutritional composition and cooking time of improved cowpea lines. Crop. Sci. 1993, 33, 469-472. [CrossRef] 
32. Boukar, O.; Fatokun, C.A.; Huynh, B.L.; Roberts, P.A.; Close, T.J. Genomic tools in cowpea breeding programs: Status and perspectives. Front. Plant Sci. 2016, 7, 757. [CrossRef] [PubMed]

33. Murdock, L.L.; Coulibaly, O.; Higgins, T.J.V.; Huesing, J.E.; Ishiyaku, M.; Sithole-Niang, I. Cowpea. In Compendium of Transgenic Crop Plants: Transgenic Legume Grains and Forages; Kole, C., Hall, T.C., Eds.; Blackwell Publishing: Oxford, UK, 2008 ; pp. 23-56.

34. Prohens, J.; Pietro, G.; Mariola, P.; Hannes, D.; Benjamin, K.; Marıa, J.D.; Ana, F.; Francisco, J.H.; Salvador, S.; Sandra, K.; et al. Introgressiomics: A new approach for using crop wild relatives in breeding for adaptation to climate change. Euphytica 2017, 213, 158. [CrossRef]

35. Raubach, S.; Kilian, B.; Dreher, K.; Amri, A.; Bassi, F.M.; Boukar, O.; Cook, D.; Cruickshank, A.; Fatokun, C.; El Haddad, N. From bits to bites: Advancement of the Germinate platform to support prebreeding informatics for crop wild relatives. Crop Sci. 2021. [CrossRef]

36. Shaw, P.; Raubach, S.; Hearne, S.; Dreher, K.; Bryan, G.; McKenzie, G.; Milne, I.; Stephen, G.; Marshall, D. Germinate 3: Development of a Common Platform to Support the Distribution of Experimental Data on Crop Wild Relatives. Crop Sci. 2017, 57, 1259-1273. [CrossRef] 